\title{
ESTUDIO DE LOS PATRONES DE MARCHA PARA UN ROBOT HEXÁPODO EN TAREAS DE BÚSQUEDA Y RESCATE
}

\author{
Jorge De León and Antonio Barrientos \\ CAR UPM-CSIC \\ jorge.deleon@upm.es, antonio.barrientos@upm.es
}

\section{Resumen}

En este artículo se profundiza en el estudio de los patrones de marcha para robots hexápodos con extremidades denominadas C-legs. En el estudio se analizan varios modos de marcha que distintos tipos de seres vivos emplean para desplazarse por el medio terrestre, y otro nuevos que se plantean al ver las limitaciones que presentan los existentes en la actualidad. Todo el estudio se ve reforzado con una serie de simulaciones realizadas donde los resultados obtenidos se analizan para seleccionar cuál es el mejor modo de marcha para una situación en concreto.

Palabras clave: Robots hexápodos, C-legs, patrones de marcha, búsqueda y rescate.

\section{Introducción}

La realización de este trabajo pretende dar un nuevo enfoque a la robótica de búsqueda y rescate (USAR), en especial a los robots terrestres que en ella se emplean.

Lamentablemente la mayoría de los robots terrestres convencionales son incapaces de lograr sus objetivos ya que su modo de locomoción, la rueda o la oruga, es incapaz de solventar los obstáculos que se presentan. El nuevo diseño que aquí se presenta pretende servir de guía para nuevos desarrollos de robots bioinspirados, y reducir, de esta forma, los porcentajes de fracaso.

De todos los robots desarrollados mediante técnica de bioinspiración, un alto porcentaje están basados en la fisionomía de los hexápodos. La clase de los hexápodos es la clasificación de los artrópodos que más especies agrupa, incluyendo en ellos a los insectos. Estos, a su vez se pueden clasificar según su sistema de locomoción sea terrestre o aéreo. Sin embargo, los robots inspirados suelen imitar a los de locomoción terrestre debido a que su adaptación al ámbito de la ingeniería es más sencillo.

El FZI (Research Center for Information Technology) en Karlsruhe, Alemania, ha creado la familia LAURON, cuyo desarrollo va por la quinta gene- ración (figura 1a). Este robot está dotado de diferentes modos de marcha y es capaz de mantener la estabilidad incluso en terrenos con numerosos obstáculos.

Otro ejemplo es el robot DIGbot, diseñado por la Case Western Reserve University, en Cleveland, Estados Unidos, y cuyo diseño de patas hace posible que pueda avanzar por troncos de árboles y paredes verticales, figura $1 \mathrm{~b}$.

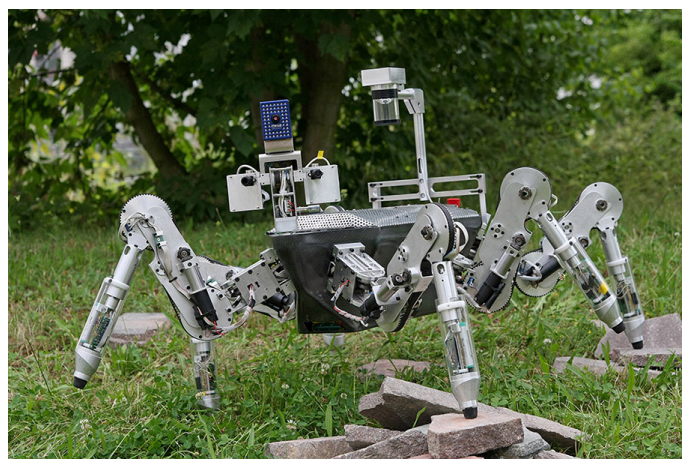

(a) Robot LAURON IV

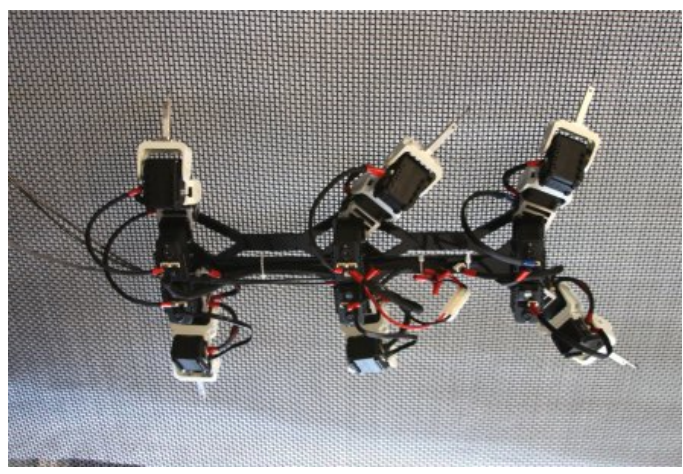

(b) Robot DIGbot

Figura 1: Ejemplos de robots hexápodos

Pero a pesar de que la cinemática de este tipo de robots está muy estudiada y resulta muy sencilla de implementar, la implementación mecánica y su control es muy complejo [6], por ello, se han desarrollado una nueva serie de robots que siguen siendo hexápodos pero presentan una nueva fisionomía en sus extremidades.

Estas nuevas extremidades tienen forma de letra $C$ y permiten a este tipo de robots desplazarse 
con unas velocidades mucho mayores y con unos requisitos mecánicos menores. Una ventaja de este tipo de robots es, entre otras, su capacidad de lo que se conoce en la literatura como "locomoción sin patas", por la cual los robots pueden superar obstáculos cuando ninguna de sus patas toca el suelo [1].

Un robot que describe este tipo de diseño es el RHex desarrollado bajo la financiación del DARPA en Estados Unidos, figura 2.

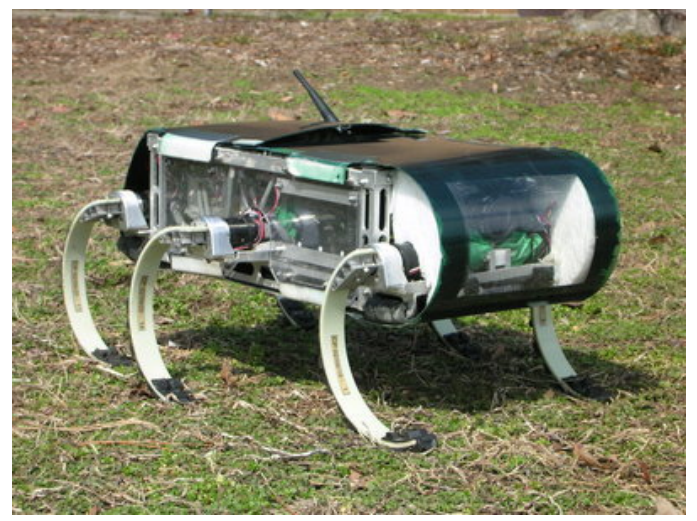

Figura 2: Robot RHex

A pesar de la gran proliferación de este tipo de robots, no se han realizado estudios que profundicen en el estudio cinemático de las patas de los mismos [4], [7], [9]. Sin embargo, este estudio es fundamental para este tipo de robots, condicionando el proceso de diseño y construcción de los mismos.

En este artículo se realiza el estudio de diversos modos de locomoción para este último tipo de robots y cuyos resultados han sido validados mediante simulaciones con un resultado correcto.

La estructura del artículo comienza con esta pequeña introducción, para continuar con el modelo del robot. Posteriormente, se procede a la definición de modos de marcha y, por último, se encuentran las pruebas y simulaciones realizadas, para terminar con las conclusiones del análisis.

\section{Modelado del robot y sus patas}

El robot que se ha empleado es un prototipo desarrollado por el grupo de Robótica y Cibernética de la Universidad Politécnica de Madrid ([7], [8]).

Este robot se ha diseñado con una serie de especificaciones para poder realizar tareas de búsqueda y rescate (USAR [5]) y también protección robotizada de infraestructuras críticas (PRIC [3]) en entornos que presentan distintos obstáculos como pueden ser edicios, complejos industriales, ... donde un robot convencional con ruedas no puede des- plazarse por todo el área a vigilar.

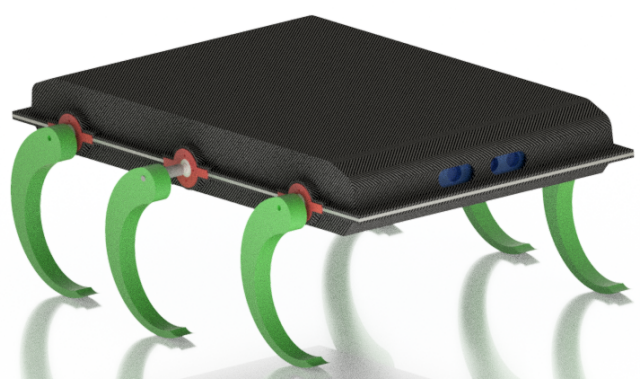

Figura 3: Render del robot diseñado

El primer requisito de diseño es poder superar una escalera, para ello según las conclusiones de los trabajos analizados es necesario que el diámetro de la pata sea igual o superior a la altura del escalón. Por lo que se ha seleccionado un tubo de acetal de $200 \mathrm{~mm}$ de diámetro, con 4,5 $\mathrm{mm}$ de espesor de pared y una anchura de $50 \mathrm{~mm}$ y que son cortados en forma de $C$.

Con esta imposición de diseño, aparece una nueva restricción, la dimensión mínima del robot, que es la suma de los tres radios de las patas más el espesor de las mismas.

$$
\begin{array}{r}
\text { largo }_{\text {robot }}>3 \cdot\left(\text { diametro }_{\text {patas }}+\text { espesor }_{\text {patas }}\right) \\
\text { largo }_{\text {robot }}>613,5 \mathrm{~mm}
\end{array}
$$

Además de estas dos restricciones, existe otra que es que pueda clasificarse como un robot manportable o man-packable.

Con esto, el diseño resultante es el que se puede observar en la figura 3.

\section{Definición y evaluación de los patrones de marcha}

Todos los hexápodos caminantes muestran la característica de ser estables; es decir que con las seis extremidades que poseen tienen buen apoyo sobre el terreno, de esta forma al retirar el apoyo de una, dos o tres extremidades, las cinco, cuatro o tres patas restantes siguen dando sustento; lo que significa que el hexápodo tiene ventajas en comparación con los bípedos y los cuadrúpedos al tener más puntos de apoyo y dar buen soporte al momento de generar los distintos modos de caminar.

\subsection{Patrones de locomoción en la naturaleza}

En base a la observación de la naturaleza, se pueden distinguir 3 tipos de marcha para los hexápo- 


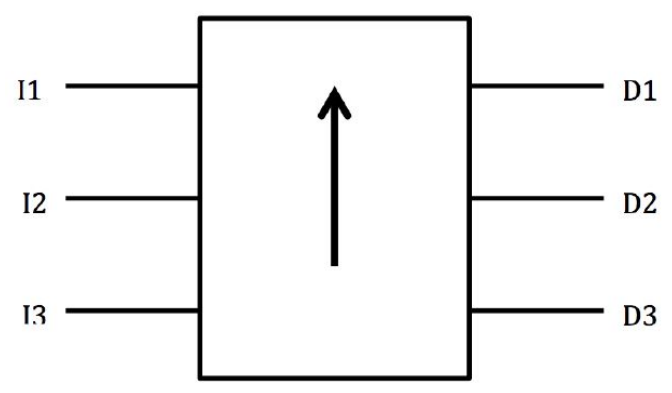

Figura 4: Esquema de la posición y definición de las patas de nuestro hexápodo

dos: de trípode alterno, tetrápoda y de onda, cuyos patrones de marcha se pueden observar en la figura 5.

Trípode alterno: Se mueven simultáneamente las extremidades I1, D2, e I3 durante un lapso de tiempo (ya sea hacia delante o hacia atrás) en el aire, área de negro; mientras que las extremidades D1, I2 y D3 permanecen en contacto con el suelo teniendo un movimiento contrario a las anteriores, área de blanco.

Tetrápoda: Se mueven por parejas de extremidades opuestas. Esto es, I1 y D3 se mueven (ya sea hacia delante o hacia atrás), áreas de negro, mientras que el resto permanece en el suelo. Dentro del lapso que dura su ciclo entra en acción la extremidad D2 de igual forma que en el anterior. A continuación las extremidades I3 y D1 hacen la misma acción que las anteriores pero durante su ciclo de trabajo. Y, por último, realiza el movimiento I2.

Onda: En este tipo de caminar las extremidades son independientes, cada una se mueve durante un lapso de tiempo (ya sea hacia delante o hacia atrás) y continúa la extremidad contigua.

\subsection{Nuevos patrones de locomoción}

Sin embargo, tal y como se ha demostrado a lo largo de la historia, el ser humano puede llegar a ser capaz de desarrollar cosas que la naturaleza no ha sido capaz. Por eso, en esta sección se van a presentar dos modos de marcha novedosos que no se han encontrado en ningún estudio biológico hasta la fecha.

El primero de ellos (modo nuevo 1) se podría definir como una variante de la marcha tetrápoda, pero en este caso la combinación de patas no presenta una transición suave. En lugar de establecer la marcha con una suave transición entre pares de
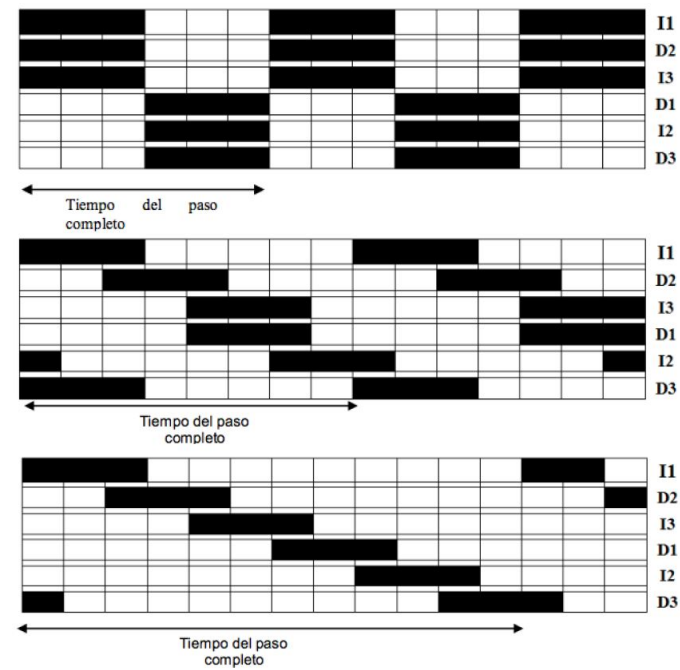

Figura 5: Modelos de marcha. (De arriba a abajo: Trípode, tetrápoda y onda)

patas, se generara un patrón de movimiento en el que en cada secuencia siempre se moverá un par de patas, primero el delantero, luego el intermedio y por último el trasero, y de nuevo volverá a empezar el patrón.

Al implementar este modo se tendrá que realizar un buen cálculo de los pares y esfuerzos generados en el par que actúa, ya que en este tipo de movimiento no se presenta un apoyo tan grande como en otros movimientos.

El segundo movimiento diseñado (modo nuevo 2) puede parecer bastante rudimentario pero no por ello dejar de ser menos efectivo, está pensado para terrenos muy irregulares. En este caso todas las patas del robot se moverán conjuntamente en cada ciclo, es decir, pasará de estar totalmente apoyado a no presentar ningún apoyo y caer sobre el suelo.

Este movimiento puede presentar el inconveniente de ser agresivo con el robot por la gran cantidad de impactos que reciba en cada transición, pero no por ello se debe dejar de plantear.
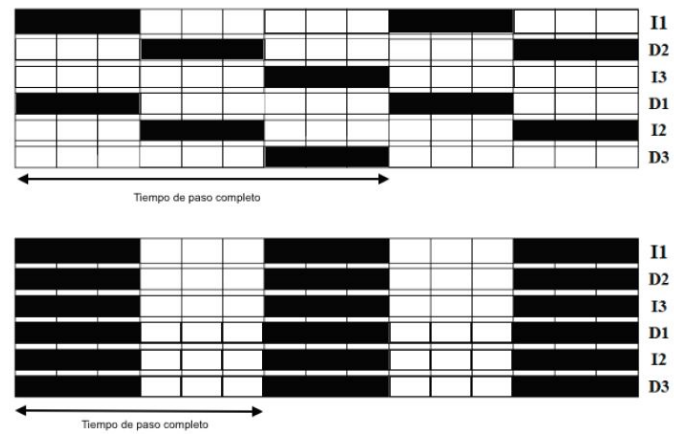

Figura 6: Nuevos modelos de marcha. 


\section{Simulaciones realizadas y resultados}

Para poder obtener un análisis completo de los modos de marcha se han realizado una serie de pruebas donde se ponen a prueba todos los modos de marcha en condiciones diferentes. Las pruebas se han realizado en la plataforma virtual de programación de robots, ROS (Robot Operating System), y el simulador Gazebo.

Como en todo proceso de diseño, la parte de simulación proporciona muchos datos de interés sobre todo para detectar posibles elementos que han sido mal diseñados (tanto de hardware como de software).

En el caso que se aborda se ha diseñado un set de cuatro pruebas donde se pondrán a prueba los algoritmos de marcha que se han programado. La primera es una prueba de desplazamiento sobre un terreno vacío, sin pendientes ni obstáculos; la segunda es una prueba de superación de una escalera; la tercera simula un terreno abrupto y la última es la superación de un plano inclinado.

Como la simulación en un ordenador se puede considerar una simulación en un entorno controlado y, por tanto, si siempre se realiza bajo las mismas condiciones, los resultados obtenidos serán idénticos, al principio de cada prueba el robot se verá afectado por una perturbación provocada adrede por el sistema. De esta forma se intenta exponer al robot a distintos casos en cada repetición de las pruebas.

Cada prueba se repetirá un total de 100 veces para tener una muestra amplia y poder obtener unos resultados más concluyentes. Además, en cada una se recogen una serie de datos para su análisis, éstos son: tiempo de la prueba, distancia recorrida, velocidad media, cuerpos/segundo, posición inicial $(\mathrm{x}, \mathrm{y})$, posición final $(\mathrm{x}, \mathrm{y}) \mathrm{y}$ altura media del centro del robot.

\subsection{Prueba 1: Desplazamiento rectilíneo}

El objetivo de la primera prueba es analizar si todos los modos de marcha son capaces de realizar un desplazamiento de por lo menos un cuerpo por segundo, lo que significaría que superan en esta habilidad a numerosos robots hexápodos ya construidos. A parte de esa cualidad, se pretende analizar cual es la velocidad máxima que es capaz de alcanzar cada modo, para lograrlo se irá aumentando sucesivamente la velocidad del robot hasta que sea incapaz de recorrer una distancia de $X$ metros con un error en el eje transversal menor al diez por ciento de la distancia recorrida (figura 7). La duración de la prueba es de 60 segundos.
En el cuadro 1 se pueden observar los resultados obtenidos.

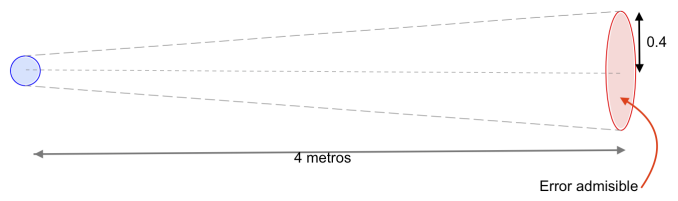

Figura 7: Error máximo permitido en la prueba 1.

Cuadro 1: Resultados de la primera prueba.

\begin{tabular}{|l|c|c|c|c|c|}
\hline Modo & Distancia $(\mathrm{m})$ & Velocidad $(\mathrm{m} / \mathrm{s})$ & Cuerpos & Pos. Final $(\mathrm{x}, \mathrm{y})$ & Altura media $(\mathrm{m})$ \\
\hline Trípode & 4,5 & 0,075 & 2,16 & $4.5 ; 0,066$ & 0,0971 \\
\hline Tetrápoda & 7,53 & 0,125 & 3,62 & $6.95 ;-2.9$ & 0,093 \\
\hline Onda & 4,45 & 0,074 & 2,13 & $-0.05 ; 4.45$ & 0,088 \\
\hline Onda V2 & 5,57 & 0,093 & 2,68 & $5.46 ; 1.12$ & 0,088 \\
\hline Nuevo Modo 1 & 4,15 & 0,069 & 1,99 & $3.98 ;-1.19$ & 0,085 \\
\hline Nuevo Modo 2 & 12,07 & 0,201 & 5,79 & $12.02 ; 1.16$ & 0,062 \\
\hline & \multicolumn{5}{|l|}{}
\end{tabular}

De la prueba número 1 se desprenden numerosos datos de interés, el primero se puede obtener del análisis de las velocidades experimentadas por el robot, el modo de marcha nuevo 1 ha triplicado en velocidad casi al resto de modos de marcha, el segundo que más velocidad media ha tenido es el modo de marcha que imita a los tetrápodos, pero muy por debajo. Aunque las velocidades puedan parecer bajas, son todo lo contrario, en robótica se considera excelente que un robot móvil pueda alcanzar una velocidad de un cuerpo por segundo. En el cuadro 2 se puede observar una comparación con el resto de robots diseñados hasta la fecha.

Cuadro 2: Comparativa de desplazamiento con otros robots.

\begin{tabular}{|l|l|l|l|l|}
\hline Robot & Longitud $(\mathrm{m})$ & Masa $(\mathrm{kg})$ & Velocidad $(\mathrm{m} / \mathrm{s})$ & Cuerpos/segundo \\
\hline CW Robot & 0,5 & 1 & 0,0833 & 0,16 \\
\hline Dante II & 3 & 770 & 0,017 & 0,006 \\
\hline Atilla & 0,36 & 2,5 & 0,03 & 0,083 \\
\hline ASV & 5 & 3200 & 1,1 & 0,22 \\
\hline Boadicea & 0,5 & 4,9 & 0,11 & 0,22 \\
\hline Sprawlita & 0,17 & 0,27 & 0,42 & 2,5 \\
\hline Robot & 0,48 & 9,5 & 12,07 & 5,79 \\
\hline
\end{tabular}

En la figura 8 se puede observar las distintas trayectorias que ha realizado cada uno de los robots. Destaca, en color blanco, el recorrido del modo 3, realizando casi un medio giro, para intentar resolver este error cometido por el patrón programado se cambio la secuencia de movimiento de las patas (figura 9) y cuyo resultado se puede ver en color amarillo.

Otro dato que se desprende de los datos es que solo el modo en trípode alterna es capaz de mantener una marcha más o menos rectilínea, el resto de modos se ven afectados por los desequilibrios que se producen en algún punto de las transiciones al no encontrarse el centro de gravedad dentro del margen de estabilidad o por el desfase que se produce en el primer paso. 


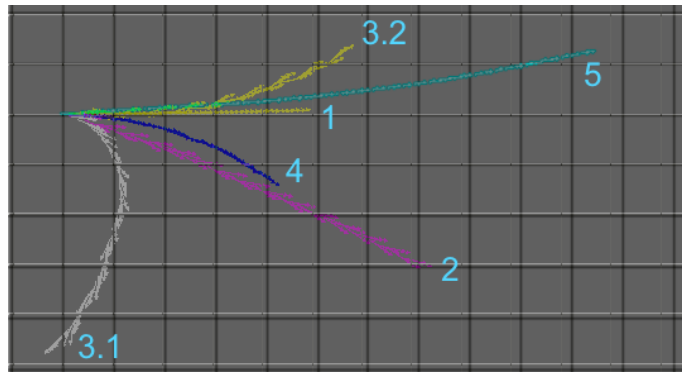

Figura 8: Trayectorias seguidas por los distintos modos de marcha.

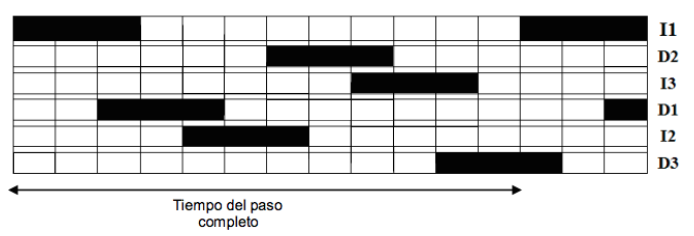

Figura 9: Nuevo modo de onda diseñado.

La comparación de la altura del centro de masas del robot también resulta interesante (figura 10). Se aprecia como algunos modos de marcha mantienen una altura más constante mientras que otros están constantemente subiendo y bajando, esto puede convertirse en un punto determinante a la hora de seleccionar el modo de marcha según la superficie donde se vaya a actuar.

\subsection{Prueba 2: Superación de una escalera}

La segunda prueba busca averiguar que tipos de marcha son capaces de superar una escalera. Esta prueba es una característica importantísima que debe tener el robot pues una de las principales limitaciones que tienen los robots USAR actuales es la incapacidad de subir escaleras, por tanto, lograr que el robot sea capaz de superar esta prueba se convierte en un objetivo esencial a superar.

La escalera diseñada para la prueba está diseñada según los estándares en arquitectura [2], donde se exige que la huella sea como mínimo de $28 \mathrm{~cm}$. y la contrahuella en el intervalo 13 a $18 \mathrm{~cm}$, cumpliendo la siguiente relación:

$54<2 \cdot$ contrahuella $+1 \cdot$ contrahuella $<70$

En el caso abordado, la contrahuella tiene una altura de $17 \mathrm{~cm}$ y la huella $34 \mathrm{~cm}$, dando un resultado de $68 \mathrm{~cm}$ (figura 11).

En este caso el modo nuevo 2 fue capaz de superar el obstáculo aunque con dificultades, el robot tenía que estar perpendicular al peldaño que iba a subir, en caso contrario no lo superaba y debía
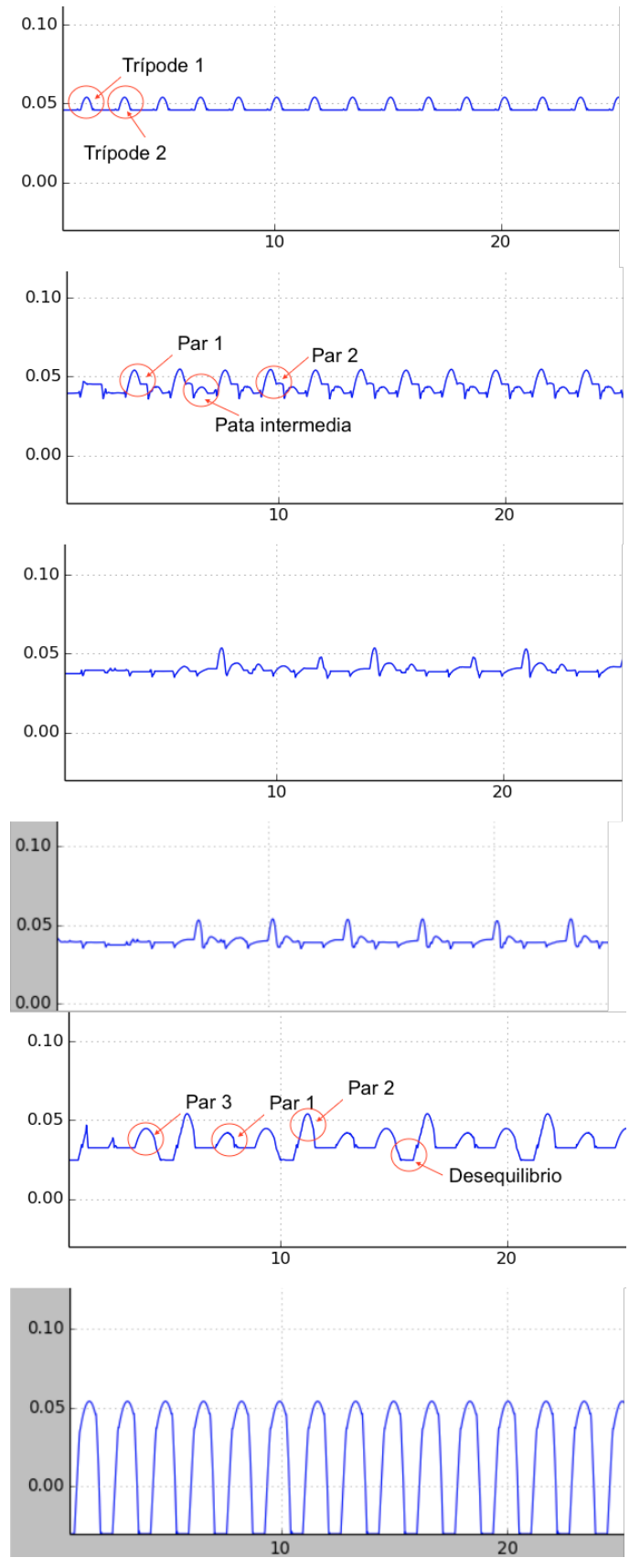

Figura 10: Altura del centro de masa en cada tipo de marcha.

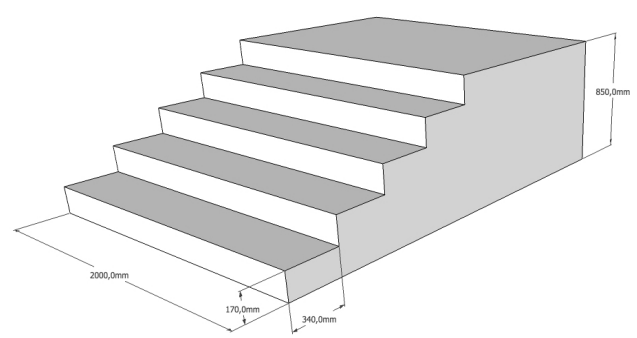

Figura 11: Modelado de la escalera para la prueba 2 . 
recolocarse, sin embargo, si no se producía ninguna desviación en la trayectoria es capaz de subir toda la escalera de una vez. También logró el objetivo el modo nuevo 1 pero siempre que estuviese colocado en la misma posición que el modo anterior y además, solo lo superar si el movimiento de las patas se realizaba par delantero. Los resultados de los dos modos que superaron la prueba se detallan en la figura 12, donde se aprecia que el modo nuevo 2 es mucho más rápido a pesar de presentar un mayor número de intentos para subir cada escalón. Por su parte, el modo nuevo 1 presenta esa lentitud debido a que en cada escalón que supera, tiene que esperar a que la secuencia de movimiento llegue de nuevo al par delantero.

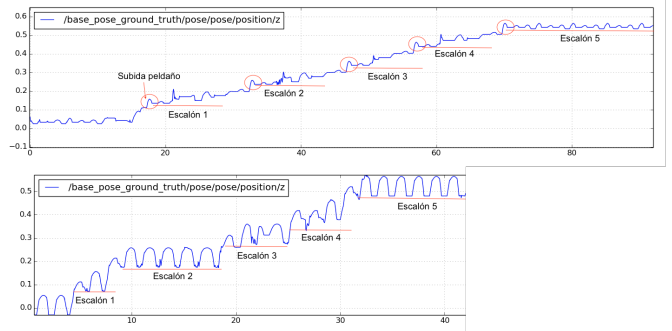

Figura 12: Resultados de la prueba 2.

Como el resto de modos de marcha seguían sin poder subir la escalera se decidió realizar un cambio en la estrategia de control, ya que uno de los motivos por los que no podían subir la escalera es la falta de par contra el escalón. Para ello, se rediseñó el algoritmo para que realizase un control de secuencias en intervalo de tiempo, el lado positivo de este control es que cuando una pata hiciese fuerza contra un escalón, transcurrido el intervalo de tiempo definido, entraría en acción la segunda pata delantera, ayudando a solventar el obstáculo. La consecuencia de emplear este modo de marcha es que se pierde la sincronización entre patas.

La conclusión de esta prueba indica claramente que la superación de obstáculos presenta dos factores claves, el primero es la relación entre el diámetro de la pata y el escalón, donde el segundo no puede ser mayor que el primero; y el segundo significa que el modo de marcha empleado debe de aplicar un par con las dos patas delanteras simultáneamente para poder superar el obstáculo.

\subsection{Prueba 3: Navegación por terreno abrupto}

Los robots USAR suelen ser diseñados para desplazarse en este tipo de terreno, pero como ya se analizó en el estado del arte, al final, un gran número son incapaces de superar este obstáculo. La prueba no trata de averiguar solo quien es capaz de superar el terreno abrupto sino también realizarlo de una manera más rápida.

El terreno modelado para la prueba se observa en la figura 13 según el modo de marcha que se seleccione el robot seguirá la trayectoria según el par que pueda aplicar en cada secuencia y las posibles colisiones entre el terreno y el cuerpo (figura 14).

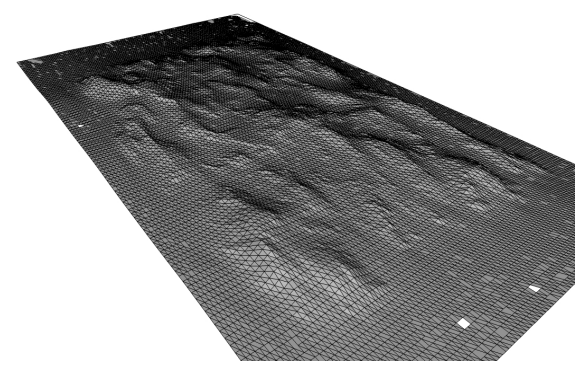

Figura 13: Modelado del terreno de la prueba 3.

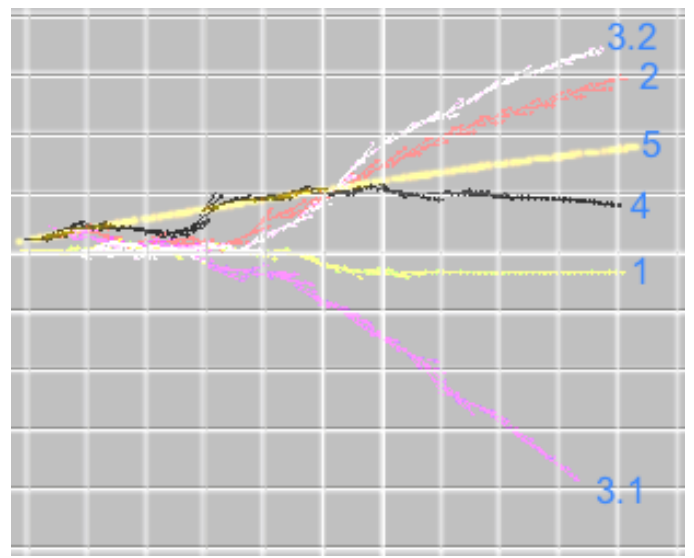

Figura 14: Resultados de la prueba 3.

Como se puede apreciar en los resultados de esta prueba, los modos de marcha de trípode alterno y el nuevo modo 1 , son los que han seguido una trayectoria más rectilínea, esto se puede deber a dos factores principales, que tienen una altura media respecto al suelo superior a los demás modos, con lo que han podido superar obstáculos que los otros modos han debido de rodear. O, que las secuencias de movimiento son capaces de generar más par para superar los obstáculos.

Por último, en la figura 15 se puede apreciar el perfil de la ruta seguida por cada modo.

\subsection{Prueba 4: Pendiente máxima}

Resulta frecuente que tras los terremotos o derrumbes de edificios, se encuentren paredes derribadas pero formando planos inclinados, o bien que haya que acceder al interior de una vivienda a través del tejado, tal y como ocurre cuando hay deslizamientos de tierra que sepultan viviendas.

Esta prueba se ha diseñado para simular estas si- 

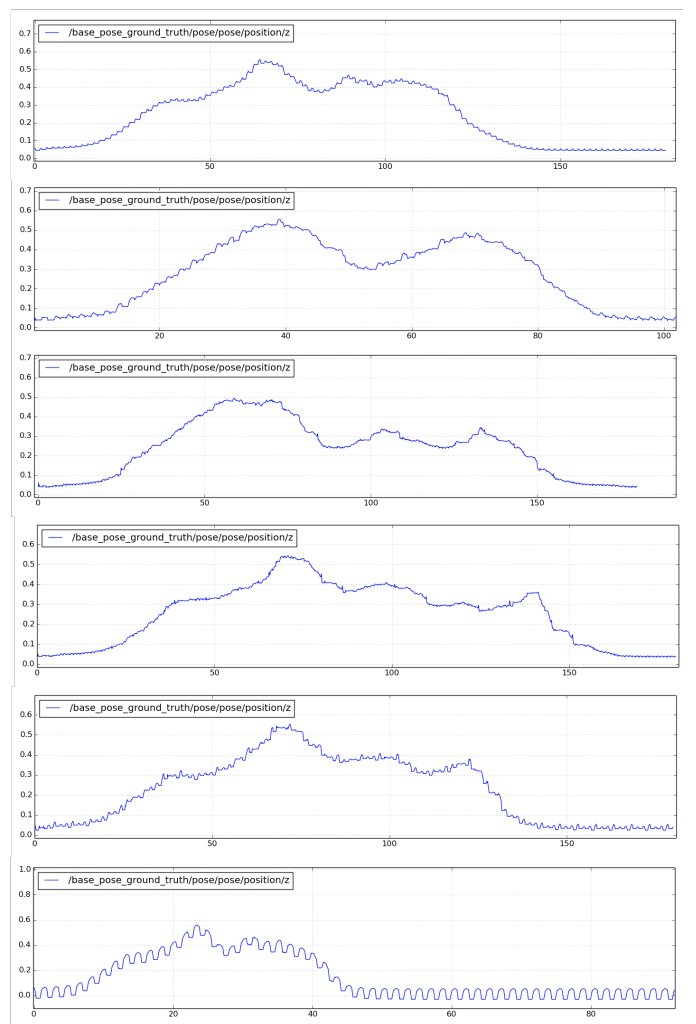

Figura 15: Perfil de la ruta seguido por cada modo de marcha en la prueba 3 .

tuaciones y averiguar que secuencia de movimientos es capaz de superar un plano más inclinado. Se empezó probando a los robots con una pendiente constante del $10 \%$ con una longitud de 4 metros en la proyección horizontal, para posteriormente ir aumentado la inclinación hasta alcanzar una pendiente máxima del $50 \%$. Los resultados obtenidos en esta prueba han sido muy satisfactorios, todos los robots han sido capaces de superarla sin ningún inconveniente. En la tabla 4.5 se muestran el tiempo empleado por cada modo en una pendiente del $50 \%$.

Cuadro 3: Resultados de superación de la prueba 4 para una pendiente del $50 \%$.

\begin{tabular}{|l|c|}
\hline Modo & Tiempo (s) \\
\hline Trípode Alterno & 80 \\
\hline Tetrápodo & 51 \\
\hline Onda & 79 \\
\hline Modo Nuevo 1 & 71 \\
\hline Modo Nuevo 2 & 20 \\
\hline
\end{tabular}

\section{Conclusiones}

Cabe resaltar los resultados obtenidos por los dos nuevos modos de marcha ideados, en especial el segundo de ellos, modo nuevo 2. Quizás hasta la fecha nadie se había planteado analizarlo por la poca naturalidad del movimiento que realiza, incluso en este trabajo se dudó desde el principio de su eficacia. Sin embargo, mucho más allá de lo esperado, ha sido con creces el movimiento que más versatilidad ha ofrecido, siendo capaz de superar todas las pruebas casi sin inconvenientes.

También se ha observado que el trípode alterno es que es capaz de realizar unas trayectorias más rectilíneas y manteniendo una altura más constante, es por ello que es el que predomina en los seres vivos hexápodos.

Sería muy interesante el poder analizar un comportamiento mixto, que en terrenos donde los obstáculos sean fáciles de esquivar o con un tamaño menor a la mitad del diámetro de la pata se emplee el trípode alterno y cuando haya que superar un obstáculo elevado se cambie el modo de marcha al modo nuevo 1 o modo nuevo 2 .

Queda, por tanto, demostrada la importancia del estudio realizado en este trabajo, abriendo nuevas vías de desarrollo a robots terrestres de rescate, donde se han superado los elementos que hasta la fecha han limitado a los robots que se desplazan mediante ruedas convencionales y orugas.

\section{Agradecimientos}

Esta investigación ha recibido fondos del proyecto RoboCity2030-III-CM (Robótica aplicada a la mejora de la calidad de vida de los ciudadanos. fase III; S2013/MIT-2748), financiado por los Programas de Actividades I+D en la Comunidad de Madrid y los Fondos Estructurales de la Unión Europea, y del proyecto DPI2014-56985-R (Protección robotizada de infraestructuras críticas), financiado por el Ministerio de Economía y Competitividad del Gobierno de España.

\section{Referencias}

[1] R. Balasubramanian. Legless locomotion: Concept and analysis. Technical report, The Robotics Institute. Carnegie Mellon University, Pittsburgh, Pennsylvania 15213, May 2004.

[2] Gobierno de Espana. Codigo tecnico de la edificacion. db-si. Technical report, Gobierno de Espana.

[3] Juan Jesús Roldán Gómez, Mario Garzón Oviedo, Jorge de León Rivas, David Alfredo Garzón Ramos, Andrés Martín Barrio, Silvia Terrile, Pablo García Auñón, Jaime Del Cerro Giner, Claudio Rossi, and Antonio Barrientos Cruz. Proyecto pric: Protec- 
ción robotizada de infraestructuras críticas. In Libro de actas de las Jornadas Nacionales de Robótica 201\%, volume 1, pages 1-6, Valencia, Junio 2017. CEA-IFAC. Robótica y Cibernética RobCib.

[4] E. Z. Moore. Leg design and stair climbing control for the rhex robotic hexapod. Master's thesis, Department of Mechanical Engineering McGill University, January 2002.

[5] Robin R. Murphy. Disaster Robotics. The MIT Press, 2014.

[6] Chenghui Nie, Xavier Pacheco Corcho, and Matthew Spenko. Robots on the move: Versatility and complexity in mobile robot locomotion. IEEE Robotics $\&$ Automation Magazine, 20(4):72/82, December 2013.

[7] Jorge De Leon Rivas. Definicion y analisis de los modos de marcha de un robot hexapodo para tareas de busqueda y rescate. Master's thesis, Escuela Superior de Ingeniera Industrial. Universidad Politecnica de Madrid, October 2015 .

[8] Jesus Tordesillas. Diseno y simulacion del sistema de locomocion de un robot hexapodo para tareas de busqueda y rescate. Master's thesis, Universidad Politecnica de Madrid, July 2016.

[9] Jesus Tordesillas, Jorge De Leon, Jaime Del Cerro, and Antonio Barrientos. Modelo cinematico de un robot con c-legs. Jornadas de Automatica, pages 267-275, September 2016. 